\title{
Analysis of the Impact of Big Data on Enterprise Decision Making
}

\author{
Guang Chen ${ }^{1, a^{*}}$, Zhanjie Liu ${ }^{1, b}$, Yixin Sun ${ }^{1, c}$, Wei Liu ${ }^{1, d}$, \\ Qiumei Lv ${ }^{1, e}$, Yukun Song ${ }^{1, f}$, He Huang ${ }^{2, g}$ and Wenjing Peng ${ }^{3, h}$ \\ ${ }^{1}$ State Grid Energy Research Institute Co. Ltd., Beijing 102209, China; \\ ${ }^{2}$ State grid information and telecommunication branch, Beijing 100761, China; \\ ${ }^{3}$ School of Economics and Management, North China Electric Power University, Beijing 102206, \\ China. \\ achenguang@sgeri.sgcc.com.cn, ${ }^{\mathrm{b}}$ liuzhanjie@sgeri.sgcc.com.cn, ${ }^{\mathrm{c}}$ sunyixin@sgeri.sgcc.com.cn \\ diuwei@sgeri.sgcc.com.cn, ${ }^{\mathrm{d}}$ Ivqiumei@sgeri.sgcc.com.cn, ${ }^{\mathrm{f}}$ songyukun@sgeri.sgcc.com.cn \\ ghuanghe@sgcc.com.cn; $358801762 @ q q . c o m$
}

*The corresponding author

Keywords: Big data; Decision basis; Decision maker; Decision environment

\begin{abstract}
The emergence and development of big data have important influence on the decision-making of enterprises. This paper analyzes the important impact of large data on enterprise decision-making from the aspects of decision-making basis, decision-making maker, decision-making power allocation, decision-making process, decision environment and so on. After that, this article also analyzes the countermeasures that the enterprise should take, and proposes that the enterprise need to accelerate the improvement and adjustment in the aspects of enterprise culture, data management, data technology and tools research and development, seize the opportunity given by the big data, and continue to improve its competitiveness so that it can gain an invincible position in the fierce market competition.
\end{abstract}

\section{Introduction}

Since 2012, the term "big data" (Big Data) has been mentioned and used more and more. People use it to describe and define the massive data generated in the era of information explosion. As time goes on, people become more and more aware of the importance of data to enterprises.

The exact definition of "large data" does not exist yet, but it is generally believed that "big data" refers to the large size of the large scale, which can not be collected, screened, analyzed and applied in a short time through the traditional software tools, so that it can not provide valuable information for the business decision.

From the concept of "big data", it also leads to the phrase "big data era". The first time that "big data era" came is a world-renowned consulting firm McKinsey. "Data has penetrated into every industry and business function area and has become an important factor of production," McKinsey said. The excavation and application of massive data foreshadowed a new wave of productivity growth and the arrival of a wave of consumer surpluses.

\section{The Impact of Large Data on Enterprise Decision Making}

Enterprise decision making is a highly complex dynamic process. Enterprises need to have corresponding data information as a support to formulate various management decisions.

In the era of big data, the emergence of big data and other technologies has made the internal and external environment of enterprise decision more and more complex and changeable. With the continuous accumulation of all kinds of uncertainty factors, enterprises have been greatly impacted by big data in decision-making basis, decision-making maker, decision-making power allocation, decision-making process and decision-making environment. Enterprise decision-making has become more and more "data driven decision-making".

The basis for decision making. In the era of big data, important changes have taken place in the decision making basis of decision makers. 
First, decision-makers shift from experience decision-making to experience decision-making and data decision making.

The decision-making basis mainly includes the experience, intuition judgment and various types of data, models and so on. The decision makers rely heavily on what kind of decision-making basis depends on whether they are perceptual decision makers or rational decision-makers. Different decision thinking modes determine the basis of decision-making for different decision makers. Perceptual decision-makers are easily affected by perceptual factors such as intuitive insight, cognitive orientation, value orientation, subjective preference and other perceptual factors [1] , while rational decision-makers have more preference for data and models.

In the era of large data, decision makers rely on empirical decision making and data decision-making by relying mainly on experience decision-making. The experience, intuition, data and model of the decision-makers complement each other, and together become the important basis for the decision of the enterprise.

Second, the position and role of data in enterprise decision making has been greatly enhanced. The proportion of data decision-making in all decision-making has risen sharply. Before the data play a "supporting role" and play an auxiliary role in the decision of the enterprise, it is now becoming the core and key of enterprise decision-making.

Third, the data sources and data types of enterprise decision making have undergone important changes. As far as data sources are concerned, enterprises have limited access to data in the past, mainly based on internal data, and the internal data are limited to financial statements and information systems. In terms of data types, the enterprise has a relatively single data type, and the main purpose is structured data and semi structured data. As far as data processing is concerned, off-line data are the most frequently processed data.

In the era of large data, enterprises can not only rely on internal data, but also can obtain massive external data, including the development of competitors, consumer preferences, the overall situation of the industry and other data information, greatly improving the scientific and reliability of enterprise decision-making. The data types faced by enterprises are increasingly complex and changeable, including both traditional structured data and semi-structured data, and unstructured data such as pictures and videos, and unstructured data are becoming the main part of the data. In addition, with the accelerated frequency of data update, enterprises have shifted from off-line analysis to real-time online processing of data.

The decision maker. First, the scope of enterprise decision-making group is gradually expanded.

Internally, full participation is an important feature of enterprise decision making. In the past, business decisions were mainly formulated by internal managers and senior experts. In the era of big data, the decision-making body of enterprises is further extended to ordinary employees and professional and technical personnel. Ordinary employees (grass-roots and frontline employees) have the convenience of obtaining business information and data, and professional technicians (such as data analysts) have the exclusive capabilities of data collection, processing and analysis. Therefore, these two groups have also become an important part of enterprise decision making. From the linear and top-down elite decision-making mode, enterprises gradually change to a non-linear, bottom-up mass decision-making mode.

Externally, enterprises increasingly attach importance to the important reference opinions provided by social organizations. In the past, corporate decisions rarely referred to the views of social organizations. In the era of big data, because of its specific advantages in database resources, data analysis technology and methods, the opinions of various "think tanks" and social organizations, such as consulting companies, universities and scientific research institutes, have been gradually paid attention to by the enterprises. Enterprises are eager to listen to the third party's objective and neutral reference opinions in the decision-making process, so as to enhance the scientific nature of enterprise decision making.

Second, the skill level of enterprise decision-makers needs to be improved.

In the past, decision makers only made decisions based on their knowledge level, experience and 
intuitive judgment. In the era of big data, decision makers also need to master the basic methods and tools of data analysis.

Third, the focus of enterprise decision makers change.

In the past, because of the scarcity of data resources and the single source channel, enterprise decision-makers put their efforts in data collection. In the era of large data, the data itself is sufficient enough, and the main body of decision can refocus its focus on finding problems, putting forward problems, identifying useful information and analyzing data in an all-round way.

The allocation of decision-making power. From the perspective of the allocation of decision-making power, the bad effect of enterprise decision-making is often attributed to the lack of proper decentralization, that is, the right to decision is not given to the appropriate participants. In the context of big data, how to configure decision power is a big challenge for business management decisions.

Enterprise decision can be divided into two modes: centralized decision and decentralized decision. Different modes show different forms of decision-making power allocation.

Chen Jianan (2010) [2] and other scholars believe that the allocation of decision-making power mainly includes three levels: the allocation of decision-making power between the enterprise and the external environment; the allocation among the internal organizations (such as the parent company and subsidiary); the allocation between the internal personnel of the enterprise and the basic units of an enterprise (department, team, etc).

Centralized decision-making means that the decision-making power is concentrated on the higher management level of the enterprise and even the highest management level. The decentralized decision means that the power is dispersed into various levels and individuals within the appropriate form, and the managers of each department have a certain autonomy [3].

It is generally believed that the decision mode adopted by an enterprise depends on factors such as the size of the enterprise, the changes of the external environment, the market competition situation, and the general degree of the products. Vicker, Meaghter and Wait and other scholars [4] research found that the larger the scale of the enterprise, the higher the uncertainty of the external environment, the greater the product customization and the more competitive market, the more the enterprises tend to take decentralization decision.

In addition, the distribution of knowledge within enterprises, knowledge transfer costs, and transfer costs of rights also affect the decision making mode [5]. The more concentrated the knowledge distribution, the higher cost of knowledge transfer and power transfer, the more advantage the enterprise has to make centralized decision making. On the contrary, it is the opposite.

In the era of big data, because the external environment of the enterprise is more and more difficult to predict, the amount of information grows exponentially, the distribution of decision knowledge is more extensive, and the requirement of decision-making is more timeliness. In addition, the enterprise changes [6] from the traditional "Pyramid" management organization structure to the flat structure, which leads to the decentralization of decision-making power within enterprises, decentralized decision making has gradually become the main form of decision making, and the development of information technology has made it possible for enterprises to decentralization decisions.

The process of decision making. Enterprises change from passive decision to advance decision.

The traditional enterprise decision making is a passive decision making and post decision making__ in the decision-making process, the enterprise often meets the problem first, and then carries out the investigation and research. Finally, the decision scheme is put forward, the timeliness of the decision is not strong, and it is easily restricted by time factors.

In the era of big data, the development of things is changing rapidly. The timeliness of enterprise decision-making is placed in an unprecedented position, and the post decision making is difficult to conform the development of the times. Enterprises are eager to achieve "decision making forward" and predict the trend of events in advance.

The global complex network authority Bala Brazil, in the the book of outbreak, put forward a 
view that the behavior of human $93 \%$ can be predicted., human daily behavior seems random and accidental, but it is easy to predict. Big data technology provides a solid foundation for enterprises to achieve "prior decision". If enterprises can build all kinds of Technology Library, model base, theme base, knowledge base and scene Library in advance, the information of market development, customer needs and competitors can be digitized, modeled and formulaic, which can make the development of things follow and realize the prejudgement of the state of affairs.

Decision-making environment. In the era of big data, the decision-making environment faced by enterprises has undergone tremendous changes.

First, the magnitude of data storage has been significantly improved. Enterprises need to increase their spending on technology research and development, equipment procurement and so on. At present, the data storage capacity of many large enterprise groups has reached the PB level and is growing at a rate of several hundred TB per year. Compared with the past, the amount of decision data that enterprises need to deal with has risen sharply. Therefore, enterprises need to invest more in information technology $\mathrm{R} \& \mathrm{D}$ and information infrastructure updating to keep pace with the development of the times.

Second, with the emergence of new technologies, the emergence of new technologies, data comprehensiveness, timeliness, and the breadth and depth of data analysis are greatly enhanced.

Data processing and analysis is the core part of enterprise decision making. Traditional data processing and analysis technology can not completely and comprehensively explore and use data, making the effectiveness of enterprise decision-making is not high. In recent years, with the emergence of advanced technologies such as large data, cloud computing, Internet of things, mobile Internet, artificial intelligence, machine learning and other advanced technologies, the integrity and timeliness of the acquisition of data has been greatly improved. By integrating multiple channels and data from different sources, the breadth and depth of enterprise decision-making have also been significantly enhanced. Through data analysis, enterprises can optimize the internal operation process, reduce costs and improve efficiency. In addition, enterprises can understand customer's consumption behavior, carry out precision marketing and improve the market share of products.

Third, the decision support system has been transformed and upgraded, in order to actively respond to the new requirements of enterprise decision under the big data environment.

The traditional decision support system has some inherent defects, for example, its application scope is limited, only a small number of people can used it, it can not adapt to the needs of full participation in decision making; the system is not open, and does not support the integration of data from other channels; it can not integrate resources such as expert system and management system in other areas, and the above system resources are also important components of decision support system.

In the era of large data, the decision support system needs to be changed and upgraded urgently. On the one hand, we should establish an open large group decision-making system structure and cooperative work model, strengthen the information communication and sharing among the group members; The other is to build a large data decision support system interface based on service, integrated intelligent analysis, quick decision analysis and independent decision function, and effectively integrate the external data information. In addition, to build a management information system with management, decision-making and prediction functions, form an enterprise decision and command platform integrating data and information collection, process control, early warning and judgment, command and coordination.

\section{Conclusion}

The arrival of the era of big data has had an important impact on enterprise decision-making. Great changes have taken place in the basis of decision making, decision-making body, decision-making power, decision-making process and decision environment.

Big data is both an opportunity and a challenge for the enterprise. Enterprises should accelerate the adjustment in the aspects of enterprise culture, data management, data technology and tools research and development, seize the opportunity, continue to improve their competitiveness, and be 
in an invincible position in the fierce market competition.

\section{Acknowledgments}

This work was supported by the science and technology project of State Grid Corporation of China, called "Research and Application of Data Mining Implementation Library on Company Operation Monitoring".

\section{References}

[1] D.H. Zhu, J. Zhang and X.F. Wang: Science and Technology Management, Vol. 34 (2013) No.4, p.172.

[2] J.A. Chen, Y.P. Li and S.T. Wu: Luojia Management Review, Vol. 01 (2010), p.137.

[3] Y.H. Lin: Technology Economy and Management Research, Vol. 04 (2003), p.31.

[4] Meagher, Kieron and Wait: Who Decides about Change and Restructuring in Organizations, Vol. 12 (2008), p.1.

[5] J. He: Technology Progress and Countermeasures, Vol. 31 (2014) No.04, p.65.

[6] X.Z. Ma and S.Q. Li: Modern Enterprise, Vol. 04 (2014), p.8.

[7] Peng L, Xiaoping Z. Social Stratification and Cooperative Behavior in Spatial Prisoners' Dilemma Games [J]. PLOS ONE, 2015, 10(7): e0131005. 